\title{
Analytical science in Switzerland and ANAKON 2011
}

\author{
Petra Dittrich • Detlef Günther • Gérard Hopfgartner • \\ Renato Zenobi
}

Published online: 31 January 2012

(C) Springer-Verlag 2012

As a tool for providing quantitative and qualitative information at the elemental or molecular level, analytical science is nowadays closely integrated with a variety of fields, for example, chemistry, biology, medicine, pharmaceutical sciences, and environmental sciences. There are two aspects of modern analytical science: the first focuses on research and development of new instrumentation and applications; the second one applies and optimizes the use of commercially available instrumentation to support environmental, biological, or medical research, in particular, in the "omics" fields. With increasing performance, lower cost, and robustness of use, analytical tools have, over the years, become a commodity, but significantly more training is required to use them efficiently. To avoid dependence on commercially driven instrument development, there is a strong need to

Published in the special issue Analytical Science in Switzerland with guest editors P. Dittrich, D. Günther, G. Hopfgartner and R. Zenobi.

\section{P. Dittrich}

Laboratory of Organic Chemistry,

Swiss Federal Institute of Technology,

Wolfgang-Pauli-Str. 10,

8093 Zürich, Switzerland

\section{Günther}

Laboratory of Inorganic Chemistry,

Swiss Federal Institute of Technology,

Wolfgang-Pauli-Str. 10,

8093 Zürich, Switzerland

G. Hopfgartner $(\bowtie)$

Life Sciences Mass Spectrometry,

University of Geneva, University of Lausanne,

Quai Ernest Ansermet 30,

CH-1211 Geneva, Switzerland

e-mail: Gerard.Hopfgartner@unige.ch

\section{R. Zenobi}

Department of Chemistry and Applied Biosciences,

Swiss Federal Institute of Technology,

8093 Zürich, Switzerland support independent fundamental research. For example, analytical tools are essential to measure and identify, in real time, molecules involved in a biological process, and mass spectrometry is a unique tool for gathering this information. Unfortunately, the analyst is very reliant on the features implemented by the manufacturers. What counts is not only the generation of large sets of data but also improving the quality and the reliability of the measurements to finally transform it into knowledge. Development of innovative analytical tools and applications has become a multidisciplinary topic of research that requires a large range of expertise involving chemistry, physics, biology, and informatics; academic institutions should still be major participants in this work. Innovative public-funded research will not only enable progress in other areas but will also drive high-level education of new young scientists.

The first part of this double special issue includes nine original papers and one review; these provide a snapshot of the breadth of analytical science performed in Switzerland, covering both fundamental aspects and applications. Interestingly, as reflected in this issue, mass spectrometry as a detector is widely available in most areas. In Switzerland, several academic institutions are contributing to education and research in analytical science. Important participants are the Universities of Applied Sciences (Eastern Switzerland, Northwestern, Sion, and Zürich), the cantonal Universities (Bern, Fribourg, Genève, Neuchâtel, Lausanne, and Zürich), and the two Swiss Federal Institutes of Technology (Lausanne and Zürich). Switzerland also has a very strong chemical and pharmaceutical industry, and private and public laboratories in which many scientists rely on analytical science in their day-to-day projects, although few research papers are published from that community despite the very high quality of the work performed.

The second part of this double special issue presents nine original papers related to research presented at ANAKON 2011. This conference, which is organized by the German, 
Swiss, and Austrian Chemical Societies, is one of the largest and most important meetings in Europe in analytical science and related areas. It was, for the first time, organized in Switzerland, on the Hönggerberg campus of the ETH Zürich (Swiss Federal Institute of Technology, Zurich). ANAKON 2011 was a big success, with over 500 scientists participating, and over 20 companies exhibiting their products and services. With sessions on separation science, miniaturization, sensors, bioanalysis, spectroscopy, nanoanalysis and imaging, elemental analysis, industrial and process analysis, environmental analysis, and instrumentation, the program was packed with state-of-the-art research and development. The articles in the "ANAKON" part of this special issue do not cover the entire spectrum of presentations given during the meeting, but give a good indication of the innovative spirit in analytical science.

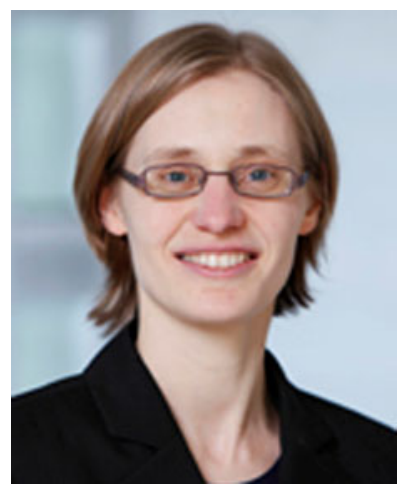

Petra Dittrich has been Assistant Professor for Bioanalysis in the Department of Chemistry and Applied Biosciences since July 2008. Her research on labon-a-chip technology focuses on miniaturization of highsensitivity devices for chemical and biological analysis, and on microfluidics-aided organization of materials. She earned her $\mathrm{PhD}$ in 2003 at the Max Planck Institute for Biophysical Chemistry (MPI Göttingen, Germany). After another year at MPI Göttingen, as a postdoctoral fellow, from 2004-2008 she worked with Andreas Manz at the Institute for Analytical Sciences (ISAS Dortmund, Germany), where she developed microfluidic techniques for cell analysis and lipid vesicle formation. She has also performed research at Cornell University (2002) and the University of Tokyo (2005). In 2008 she obtained an ERC Starting Grant for research on biomimetic membrane formation and on micro and nanofluidic manipulation. She was awarded the "Analytica Forschungspreis" in 2010.

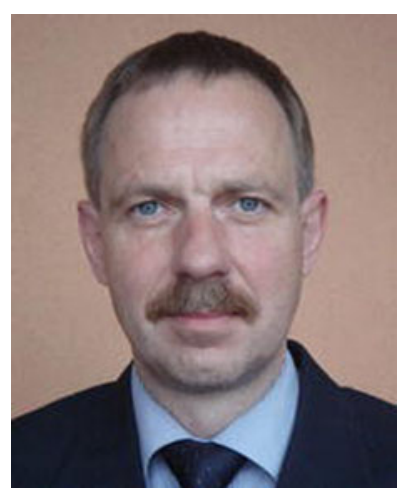

Detlef Günther is Full Professor for Trace Element and Micro Analysis in the Laboratory of Inorganic Chemistry at ETH Zurich and is currently Chair of the Department for Chemistry and Applied Biosciences. His research program focuses on fundamental and applied studies in inductively coupled plasmamass spectrometry (ICP-MS) and laser ablation-inductively coupled plasma-mass spectrometry (LA-ICP-MS), which includes studies on laser-sample interaction, aerosol transport, and plasma-related excitation processes. His fundamental understanding of UV-ns and UV-fs laser ablation in combination with Q-ICP-MS, SF-ICP-MS, ICP-TOFMS, and also MCICP-MS, and alternative excitation sources has been demonstrated in a wide variety of applications, e.g., analysis of fluid inclusions, gemstones, metals, minerals, ceramics, and other industrial materials.

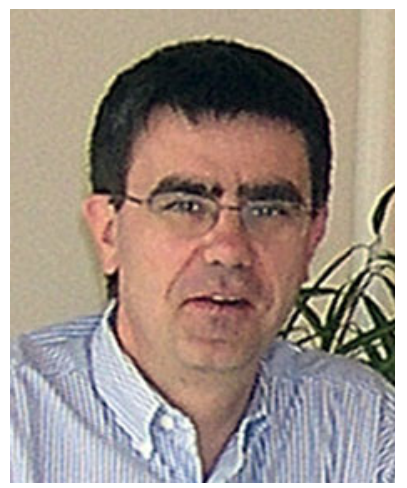

Gérard Hopfgartner is Full Professor of Analytical Pharmaceutical Chemistry and Mass Spectrometry in the Laboratory of Life Sciences Mass Spectrometry at the School of Pharmaceutical Sciences of the University of Lausanne and Geneva. His scientific interests focus on the development and application of novel mass spectrometry approaches (from QqQ $\mathrm{LIT}_{\text {to }}$ FT-ICR, including ion mobility) with and without chromatography, in the life sciences. His research is based on novel qualitative and quantitative methods for low-molecularweight compounds, peptides, and proteins in analytical metabolomics, proteomics, mass spectrometry imaging, drug metabolism, and toxicology.

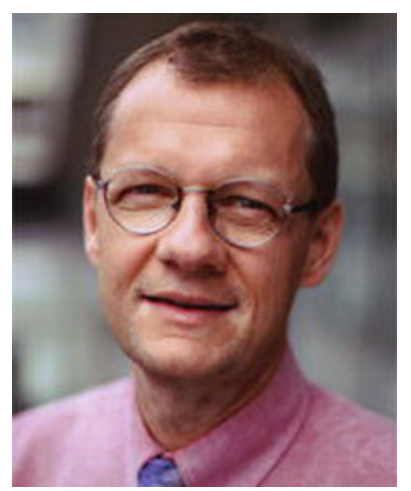

Renato Zenobi is Professor of Analytical Chemistry at the Organic Chemistry Laboratory of the Swiss Federal Institute of Technology (ETH), Zurich. He was also one of the founders and directors of the center of excellence in analytical chemistry (CEAC, 19952007) at ETH Zurich. In 2010 he was appointed Associate Editor of Analytical Chemistry (American Chemical Society). Zenobi's research includes laser-based analytical chemistry, electrospray and laser-assisted mass spectrometry, lasersurface interactions, and near-field optical microscopy and spectroscopy. He has made important contributions to understanding the mechanism of ion formation in matrix-assisted laser desorption/ionization (MALDI) mass spectrometry, to ambient ionization methods, and to the development of tools for nanoscale analysis. Renato Zenobi has received many honors for his scientific work, among them the Ruzicka Prize and the Heinrich Emanuel Merck Prize, and honorary professorships from several places including the Chinese Academy of Sciences (Changchun). 